\title{
On Some Correspondence between \\ Holomorphic Functions in the Unit Disc and Holomorphic Functions in the Left Halfplane \\ by
}

Ewa LIGOCKA

Presented by Józef SICIAK

Summary. We study a correspondence $L$ between some classes of functions holomorphic in the unit disc and functions holomorphic in the left halfplane. This correspondence is such that for every $f$ and $w \in \mathbb{H}$, $\exp (L(f)(w))=f(\exp w)$.

In particular, we prove that the famous class $S$ of univalent functions on the unit disc is homeomorphic via $L$ to the class $S(\mathbb{H})$ of all univalent functions $g$ on $\mathbb{H}$ for which $g(w+2 \pi i)=g(w)+2 \pi i$ and $\lim _{\operatorname{Re} z \rightarrow-\infty}(g(w)-w)=0$.

1. Introduction and preliminaries. A usual way to establish a correspondence between holomorphic functions in the left halfplane and those in the unit disc is to take the composition with the fractional linear map $\varphi(z)=\frac{z+1}{z-1}$. Note that $\varphi \circ \varphi=\mathrm{Id}$.

In this note we shall use the fact that the exponential function $\exp (z)=$ $e^{z}$ is a covering map from the left halfplane onto the punctured unit disc to define another correspondence between some classes of holomorphic functions.

Let us introduce some notations. $\mathbb{D}$ will denote the unit disc and $\mathbb{H}$ will denote the left halfplane $\{z: \operatorname{Re} z<0\}$. Let $\ln$ stand for the branch of the logarithm such that $-\pi<\operatorname{Im} \ln z \leq \pi$ and $\ln 1=0$.

We now define some classes of functions holomorphic on $\mathbb{D}$ or $\mathbb{H}$.

1) $S_{0}=\{f \in H(\mathbb{D}): f(0)=0$ and $f(z) \neq 0$ for $z \neq 0\}$.

2010 Mathematics Subject Classification: Primary 30C45.

Key words and phrases: holomorphic functions, univalent functions, class $S$, exponential function, logarithm. 
2) $S_{k}$ consists of the functions from $S_{0}$ which have a zero of order $k$ at zero.

Each function from $S_{k}$ can be written as

$$
f(z)=c z^{k} f_{1}(z), \quad \text { where } c \neq 0, f_{1}(0)=1
$$

and $f_{1}(z)$ does not vanish on $\mathbb{D}$. We have $S_{0}=\bigcup_{k=1}^{\infty} S_{k}$.

3) $S_{k}^{1}$ consists of the functions from $S_{k}$ for which $c=1$.

4) $S$ contains all functions from $S_{1}^{1}$ which are univalent on $\mathbb{D}$. In other words, it is the class of functions $f$ univalent on $\mathbb{D}$ and such that $f(0)=0$ and $f^{\prime}(0)=1$. This is the most important of all the classes considered. Note that $f(z)=z+z^{2}$ belongs to $S_{1}^{1}$ but not to $S$.

5) $S_{0}(\mathbb{H})$ consists of the functions $h$ holomorphic on $\mathbb{H}$ for which there exist $k \in \mathbb{N}$ and $a \in \mathbb{C}$ with $-\pi<\operatorname{Im} a \leq \pi$ such that

(i) $h(w)-k w$ is a $2 \pi i$-periodic function,

(ii) if $\left\{w_{n}\right\}_{n \in \mathbb{N}}$ is a sequence in $\mathbb{H}$ for which $\lim _{n \rightarrow \infty} \operatorname{Re} w_{n}=-\infty$ then

$$
\lim _{n \rightarrow \infty}\left(h\left(w_{n}\right)-k w_{n}\right)=a .
$$

6) $S_{k}(\mathbb{H})$ consists of all functions from $S_{0}(\mathbb{H})$ for which (i) and (ii) hold with the given $k \in \mathbb{N}$.

7) $S_{k}^{0}(\mathbb{H})$ consists of all functions from $S_{k}(\mathbb{H})$ for which $a=0$.

8) $S(\mathbb{H})$ is the class of univalent functions from $S_{1}^{0}(\mathbb{H})$.

If $h \in S_{k}(\mathbb{H})$ then $h(w+2 \pi i)=h(w)+2 k \pi i$.

In particular, if $h \in S_{1}(\mathbb{H})$ then

$$
h(z+2 \pi i)=h(z)+2 \pi i .
$$

Note that all sets $S_{k}(\mathbb{H})$ and $S_{k}^{0}(\mathbb{H})$ are convex.

2. The correspondence. Let $f \in S_{0}$. Then $f \in S_{k}$ for some $k \in \mathbb{N}$, and $f$ can be written as $f(z)=c z^{k} f_{1}(z), c \neq 0, f_{1}(0)=1$. By a monodromy argument there exists $g$ holomorphic on $\mathbb{D}$ with $g(0)=0$ such that $f_{1}(z)=$ $e^{g(z)}$. For $w \in \mathbb{H}$ put

$$
L(f)(w):=\ln c+k w+g\left(e^{w}\right) .
$$

It is easy to check that $L(f)(w) \in S_{0}(\mathbb{H})$. Since $e^{w}$ is $2 \pi i$-periodic, (i) holds. Condition (ii) is satisfied because if $\operatorname{Re} w_{n} \rightarrow-\infty$ then $e^{w_{n}} \rightarrow 0$ and $g\left(e^{w_{n}}\right) \rightarrow 0$. We have

$$
e^{L(f)(w)}=f\left(e^{w}\right) \quad \text { for each } w \in \mathbb{H} .
$$

TheOREM 1. For each $k \in \mathbb{N}$ the mapping $L$ is one-to-one and maps $S_{k}$ onto $S_{k}(\mathbb{H})$. It also maps $S_{k}^{1}$ onto $S_{k}^{0}(\mathbb{H})$.

Proof. The fact that $L$ is one-to-one follows directly from $(*)$. If $f_{1}, f_{2} \in$ $S_{k}$ and $L\left(f_{1}\right)=L\left(f_{2}\right)$ then $f_{1}\left(e^{w}\right)=f_{2}\left(e^{w}\right)$ for each $w \in \mathbb{H}$, and so $f_{1}=f_{2}$. 
Now let $h \in S_{k}(\mathbb{H})$. Put $g_{1}(w)=h(w)-k w-a$. If $z \in \mathbb{D}, z \neq 0$, then there exists $w \in \mathbb{H}$ such that $e^{w}=z$. Define $g(z)=g_{1}(w)$. Since $g_{1}$ is $2 \pi i$-periodic, $g$ is well defined on $\mathbb{D} \backslash\{0\}$. We have $g(z)=g_{1}(\ln z)$ for each branch of $\ln z$. Since $\exp (z)=e^{z}$ is a covering map, $g(z)$ is holomorphic on $\mathbb{D} \backslash\{0\}$. Condition (ii) implies that $g_{1}(w) \rightarrow 0$ if $\operatorname{Re} w \rightarrow-\infty$. Hence $g(z) \rightarrow 0$ for $z \rightarrow 0$. Thus $g(z)$ extends to a function holomorphic on $\mathbb{D}$ by setting $g(0)=0$. Now define $f(z)=e^{a} z^{k} e^{g(z)}$. Then $f \in S_{k}$ and $L(f)=h$.

It also follows from the above proof that $L$ maps $S_{k}^{1}$ onto $S_{k}^{0}(\mathbb{H})$.

Let now $H(\mathbb{D})$ and $H(\mathbb{H})$ be the spaces of holomorphic functions on $\mathbb{D}$ and $\mathbb{H}$ respectively, endowed with the compact-open topology. We shall consider $S_{k}^{1}$ and $S_{k}^{0}(\mathbb{H})$ as topological subspaces of $H(\mathbb{D})$ and $H(\mathbb{H})$ respectively.

We have

THEOREM 2. The mapping $L$ is a homeomorphism between $S_{k}^{1}$ and $S_{k}^{0}(\mathbb{H})$ for each $k \in \mathbb{N}$.

Proof. Let $\left\{f_{n}\right\}_{n \in \mathbb{N}} \subset S_{k}^{1}$ converge almost uniformly to $f_{0} \in S_{k}^{1}$. We have $f_{n}(z)=z^{k} e^{g_{n}(z)}, g_{n}(0)=0$ for each $n \in \mathbb{N}$ and $f_{0}(z)=z^{k} e^{g_{0}(z)}$, $g_{0}(0)=0$. The almost uniform convergence of $f_{n}$ implies that $e^{g_{n}}$ converges almost uniformly to $e^{g_{0}}$. Since $g_{n}^{\prime}=\left(e^{g_{n}}\right)^{\prime} / e^{g_{n}}$ and $g_{0}^{\prime}=\left(e^{g_{0}}\right)^{\prime} / e^{g_{0}}$ we see that $g_{n}^{\prime}$ converges almost uniformly to $g_{0}^{\prime}$. Since $g_{n}(0)=g_{0}(0)=0$ for all $n$, we have $g_{n}(z)=\int_{0}^{z} g_{n}^{\prime}(\xi) d \xi$. Thus $g_{n}$ converges almost uniformly to $g_{0}$. It follows that $L\left(f_{n}\right)(w)=k w+g_{n}\left(e^{w}\right)$ converges almost uniformly on $\mathbb{H}$ to $L\left(f_{0}\right)(w)=k w+g_{0}\left(e^{w}\right)$.

Conversely, if $L\left(f_{n}\right)$ converges almost uniformly to $L\left(f_{0}\right)$ on $\mathbb{H}$ then $g_{n}$ converges almost uniformly to $g_{0}$ and hence $f_{n}$ converges almost uniformly to $f_{0}$.

WARning. The mapping $L$ is not continuous between $S_{k}$ and $S_{k}(\mathbb{H})$ (with compact-open topology) (because of the term $a=\ln c$ in the definition of $L)$.

We can also prove

Proposition 1. The class $S_{k}^{1}$ is a closed subset of $H(\mathbb{D})$, and $S_{k}^{0}(\mathbb{H})$ is a closed subset of $H(\mathbb{H})$.

Proof. The Hurwitz theorem implies that $S_{k}^{1}$ is a closed subset of $H(\mathbb{D})$. If the sequence $h_{n}(w)=L\left(f_{n}\right)(w)$ converges almost uniformly on $\mathbb{H}$ then the sequence $g_{n}\left(e^{w}\right)$ converges almost uniformly on $\mathbb{H}$ since for $f_{n}=z^{k} e^{g_{n}(z)}$ with $g_{n}(0)=0$ we have $h_{n}(w)=k w+g_{n}\left(e^{w}\right)$. Then $g_{n}$ converges almost uniformly on $\mathbb{D} \backslash\{0\}$ and therefore on $\mathbb{D}$. It follows that $f_{n}$ converges on $\mathbb{D}$ to $f_{0} \in S_{k}^{1}$. We have $L\left(f_{0}\right)=\lim _{n \rightarrow \infty} h_{n} \in S_{k}^{0}(\mathbb{H})$.

REMARK 1. The assumptions $g(0)=0$ and $-\pi<\operatorname{Im} a \leq \pi$ were introduced to ensure that $L$ is a one-to-one correspondence between $S_{0}$ 
and $S_{0}(\mathbb{H})$. If we omit them we obtain a $1-\infty$ correspondence. For every $f \in S_{0}$ we shall have a countable family of functions $\left\{L_{m}(f)\right\}_{m \in \mathbb{Z}}$, $L_{m}(f)=L(f)+2 m \pi i$.

REMARK 2. Let $f \in S_{k}$ and let

$$
f_{m}:=\sqrt[m]{f\left(z^{m}\right)}=c^{1 / m} \cdot z^{k} \cdot e^{g\left(z^{m}\right) / m} \quad \text { for } m \in \mathbb{N} .
$$

Then

$$
L\left(f_{m}\right)(w)=\frac{\ln c}{m}+k w+\frac{1}{m} g\left(e^{m w}\right) .
$$

REMARK 3. The correspondence $L$ can be used to construct other classes of holomorphic functions. Let $\varphi(z)=\frac{z+1}{z-1}$. Let $f \in S_{1}$. We have $L(f)(w+$ $2 \pi i)=L(f)(w)+2 \pi i$. Put $\Lambda(f)=\varphi \circ L(f) \circ \varphi$. The function $\Lambda(f)$ maps $\mathbb{D}$ into the Riemann sphere $\widehat{\mathbb{C}}$ and has the following properties.

1) The nontangential limit of $\Lambda(f)$ at 1 is equal to 1 .

2) We have

$$
\begin{aligned}
& \forall_{k \in \mathbb{Z}} \quad u_{k} \circ \Lambda(f)=\Lambda(f) \circ u_{k}, \quad u_{0}=\mathrm{Id}, \\
& u_{k}(z)=\frac{\bar{a}_{k}}{a_{k}} \cdot \frac{z-a_{k}}{1-\bar{a}_{k} z}, \quad a_{k}=\frac{k \pi i}{1+k \pi i} \quad \text { if } k \neq 0 .
\end{aligned}
$$

For $f(z)=z e^{g(z)}$ with $g(0)=0$,

$$
\Lambda(f)(z)=\frac{2 z+g\left(e^{\frac{z+1}{z-1}}\right) \cdot(z-1)}{2+g\left(e^{\frac{z+1}{z-1}}\right) \cdot(z-1)} .
$$

3. The case of univalent functions. We start from

Theorem 3. Let $f \in S_{1}$. The function $f$ is univalent iff $L(f)$ is univalent.

Proof. Let $f=c z e^{g(z)} \in S_{1}$. Assume that $f$ is univalent. Let $L(f)\left(w_{1}\right)=$ $L(f)\left(w_{2}\right)$. Since $f$ is univalent and $e^{L(f)(w)}=f\left(e^{w}\right)$, we see that $e^{w_{1}}=e^{w_{2}}$ and $w_{1}=w_{2}+2 m \pi i$ for some $m \in \mathbb{Z}$.

We have $L(f)(w)=\ln c+w+g\left(e^{w}\right)$. Hence $L(f)\left(w_{1}\right)=L(f)\left(w_{2}\right)$ and $w_{1}=w_{2}+2 m \pi i$ imply that $m=0$ and $w_{1}=w_{2}$.

Assume now that $L(f)$ is univalent. Since $f \in S_{1}$ we have $L(f)(w+2 \pi i)$ $=L(w)+2 \pi i$. Assume that $f\left(z_{1}\right)=f\left(z_{2}\right)$. If it is equal to zero then $z_{1}=z_{2}$ $=0$ by the definition of $S_{1}$. Hence we can assume that there exist $w_{1}, w_{2} \in \mathbb{H}$ such that $z_{1}=e^{w_{1}}$ and $z_{2}=e^{w_{2}}$. This implies, as before, that $e^{L(f)\left(w_{1}\right)}=$ $e^{L(f)\left(w_{2}\right)}$, so there exists $m \in \mathbb{Z}$ for which $L(f)\left(w_{1}\right)=L(f)\left(w_{2}\right)+2 m \pi i=$ $L(f)\left(w_{2}+2 m \pi i\right)$. Thus $w_{1}=w_{2}+2 m \pi i$ and $e^{w_{1}}=e^{w_{2}}$. Hence $z_{1}=z_{2}$.

Theorem 3 is not true for $S_{k}$ with $k>1$. The function $f(z)=z^{k}, k>1$, is not univalent but $L(f)(w)=k w$ is univalent.

Proposition 2. For every $f \in S_{k}$ there exists $f_{1} \in S_{1}$ such that $f=f_{1}^{k}$. 
Proof. For $f(z)=c z^{k} e^{g(z)}$ take $f_{1}(z)=c^{1 / k} z e^{g(z) / k}$.

Theorem 3 and Proposition 2 yield

Theorem $3^{\prime}$. Let $f \in S_{k}$. The function $L(f)$ is univalent iff $f=f_{1}^{k}$ where $f_{1} \in S_{1}$ is univalent.

Proof. There exists $m \in \mathbb{Z}$ such that

$$
L(f)=L\left(f_{1}^{k}\right)=k \cdot L\left(f_{1}\right)+2 m \pi i .
$$

EXAMPLES.

1. Let $f(z)=z+z^{2} / 2$. Then $f \in S_{1}$ is univalent and hence $L(f)(w)=$ $w+\ln \left(1+e^{w} / 2\right)$ is univalent on $\mathbb{H}$.

2. If $f(z)=z+z^{2}$ then $L(f)(w)=w+\ln \left(1+e^{w}\right)$. The function $L(f)$ is not univalent because $f$ is not.

In the rest of this note we shall study the famous class $S$ of univalent functions from $S_{1}^{1}$.

Let us consider $S$ as a subset of $H(\mathbb{D})$ with compact-open topology and $S(\mathbb{H})$ as a subset of $H(\mathbb{H})$ with compact-open topology. Recall that $S(\mathbb{H})$ is the set of univalent functions from $S_{1}^{0}(\mathbb{H})$.

The Hurwitz theorem implies that $S$ is closed in $H(\mathbb{D})$. Proposition 1 together with Theorems 1, 2 and 3 shows that $S(\mathbb{H})$ is closed in $H(\mathbb{H})$.

As an immediate consequence of Theorem 3 we have

TheOREM 4. The mapping $L$ is a homeomorphism from $S$ onto $S(\mathbb{H})$.

Corollary. The class $S(\mathbb{H})$ is compact in $H(\mathbb{H})$. More generally, for each $k \in \mathbb{N}$ the class $\widetilde{S}_{k}^{0}(\mathbb{H})$ consisting of all univalent functions from $S_{k}^{0}(\mathbb{H})$ is compact in $H(\mathbb{H})$.

Proof. The class $S$ is compact since for each $f \in S$ and $z \in \mathbb{D},|f(z)| \leq$ $\sum_{n=1}^{\infty} n|z|^{n}=|z| /(1-|z|)^{2}$ (De Branges Theorem). Thus $S(\mathbb{H})$ must also be compact. Moreover, by Theorem $3^{\prime}, \widetilde{S}_{k}^{0}(\mathbb{H})$ is the continuous image of $S$ under the mapping $f \mapsto L\left(f^{k}\right)$. Hence $\widetilde{S}_{k}^{0}(\mathbb{H})$ is compact.

REMARK 4. The class $S_{1}^{0}(\mathbb{H})$ is not compact since $S_{1}^{1}$ is not compact. It contains all functions $f_{c}(z)=z e^{c z}, c \in \mathbb{C}$. The set of values $f_{c}^{\prime \prime}(0)=2 c$ is not bounded.

We now consider two important definitions:

Definition 1. A univalent function $f \in H(\mathbb{D})$ with $f(0)=0$ is starlike iff $f(\mathbb{D})$ is a domain starlike with respect to zero.

Definition 2. A univalent function $f \in H(\mathbb{D})$ with $f(0)=0$ is convex iff $f(\mathbb{D})$ is a convex domain. 
The following facts are well known and can be found in $[\mathrm{D}]$ or $[\mathrm{P}]$.

Theorem A. A function $f$ is starlike iff $\operatorname{Re}\left\{z f^{\prime}(z) / f(z)\right\}>0$.

TheOREM B. A function $f$ is convex iff the function $f_{1}(z)=z f^{\prime}(z)$ is starlike. (One assumes here that $f \in H(\mathbb{D})$ and $f(0)=0$.)

THEOREM 5.

(1) $f \in S$ is starlike iff $\operatorname{Re}(L(f))^{\prime}(w)>0$ for all $w \in \mathbb{H}$.

(2) $f \in S$ is convex iff

$$
\forall_{w \in \mathbb{H}} \quad \operatorname{Re}\left((L(f))^{\prime}(w)+\frac{(L(f))^{\prime \prime}(w)}{(L(f))^{\prime}(w)}\right)>0 .
$$

Proof. (1) Let $f(z)=z e^{g(z)}$. We have

$$
\frac{z f^{\prime}(z)}{f(z)}=\frac{z\left(e^{g(z)}+z e^{g(z)} g^{\prime}(z)\right)}{z e^{g(z)}}=1+z g^{\prime}(z)
$$

$L(f)(w)=w+g\left(e^{w}\right)$ and $(L(f))^{\prime}(w)=1+g^{\prime}\left(e^{w}\right) e^{w}=1+z g^{\prime}(z)$ for $z=e^{w}$. If $z=0$ then $z f^{\prime}(z) / f(z)=1$. Hence and by Theorem A, $f$ is starlike iff $\operatorname{Re}(L(f))^{\prime}(w)>0$.

(2) By Theorem B, $f(z)=z e^{g(z)}$ is convex iff $f_{1}(z)=z f^{\prime}(z)=z\left(e^{g(z)}+\right.$ $\left.z e^{g(z)} g^{\prime}(z)\right)=z e^{g(z)+\ln \left(1+z g^{\prime}(z)\right)}$ is starlike. Note that $\operatorname{Re}\left(1+z g^{\prime}(z)\right)>0$ for $z \in \mathbb{D}$.

Hence and by the first part of Theorem 5, $f$ is convex iff $\operatorname{Re}\left(L\left(f_{1}\right)\right)^{\prime}(w)$ $>0$ for each $w \in \mathbb{H}$. We have

$$
L\left(f_{1}\right)=L(f)+\ln \left((L(f))^{\prime}(w)\right)
$$

since $L\left(f_{1}\right)(w)=w+g\left(e^{w}\right)+\ln \left(1+e^{w} g^{\prime}\left(e^{w}\right)\right)$. We obtain

$$
\left(L\left(f_{1}\right)\right)^{\prime}=(L(f))^{\prime}+\frac{(L(f))^{\prime \prime}}{(L(f))^{\prime}} \text {. }
$$

Thus $f$ is convex iff

$$
\forall_{w \in \mathbb{H}} \quad \operatorname{Re}\left(L\left(f_{1}\right)\right)^{\prime}(w)=\operatorname{Re}\left((L(f))^{\prime}(w)+\frac{(L(f))^{\prime \prime}(w)}{(L(f))^{\prime}(w)}\right)>0 .
$$

REMARK. We thank the referee for pointing out that the operator very similar to our $L$ was used in Krzyż's paper [K, proof of Theorem 1] to study quasiconformal automorphisms of the unit disc. Very recently Chéritat [Ch] used Krzyż's operator to construct a holomorphic function with a strange Siegel disc.

\section{References}

[Ch] A. Chéritat, Relatively compact Siegel discs with non-locally connected boundaries, arXiv:0906.1048, 2009. 
[D] P. L. Duren, Univalent Functions, Springer, New York, 1983.

[K] J. Krzyż, Quasicircles and harmonic measure, Ann. Acad. Sci. Fenn. Ser. AI Math. 12 (1987), 19-24.

[P] Ch. Pommerenke, Univalent Functions, Vandenhoeck and Ruprecht, Göttingen, 1975.

Ewa Ligocka

Pasteura 4/6 m. 31

02-093 Warszawa, Poland

E-mail: elig@mimuw.edu.pl

Received April 21, 2009;

received in final form October 20, 2009 